\title{
All-space existence and dispersion of athermal directions in monoclinic $\mathrm{KY}\left(\mathrm{WO}_{4}\right)_{2}$
}

\author{
P.A. Loiko ${ }^{a}$, V.V. Filippov ${ }^{b}$, N.V. Kuleshov ${ }^{a}$, A.A. Pavlyuk ${ }^{c}$, K.V. Yumashev ${ }^{\text {a,* }}$ \\ ${ }^{a}$ Center for Optical Materials and Technologies, Belarusian National Technical University, 220013 Minsk, 65/17 Nezavisimosti Ave, Belarus \\ ${ }^{\mathrm{b}}$ B.I. Stepanov Institute of Physics, National Academy of Sciences of Belarus, 220072 Minsk, 68 Nezavisimosti Ave, Belarus \\ c A.V. Nikolaev Institute of Inorganic Chemistry, Siberian Branch of Russian Academy of Sciences, 630090 Novosibirsk, 3 Lavrentyev Ave, Russia
}

\section{A R T I C L E I N F O}

\section{Article history:}

Received 6 March 2014

Received in revised form

6 April 2014

Accepted 16 April 2014

Available online 29 April 2014

Keywords:

Double tungstates

Athermal behavior

Dispersion

Optical axis

\begin{abstract}
A B S T R A C T
The analytical expressions for thermo-optic coefficients, $\mathrm{d} n / \mathrm{d} T$, for "fast" and "slow" light waves propagating along the arbitrary direction in a biaxial crystal are derived. On the basis of these expressions, the all-space analysis of existence of athermal directions is performed for monoclinic and biaxial $\mathrm{KY}\left(\mathrm{WO}_{4}\right)_{2}$ at $1.03 \mu \mathrm{m}$. The calculations are performed for an arbitrary light propagation direction and polarization (not restricted to the principal planes). The appearance of directions that can be athermal for both "fast" and "slow" waves is predicted. The dispersion of athermal directions is analyzed for visible and near-IR. The existence of upper and lower dispersion limits for athermal behavior of KY $\left(\mathrm{WO}_{4}\right)_{2}$ is shown. It is shown that the optical indicatrix axes can be athermal itself at some light wavelengths. Wavelength- and temperature-dependent position of the optical axes of $\mathrm{KY}\left(\mathrm{WO}_{4}\right)_{2}$ is also determined.
\end{abstract}

(c) 2014 Elsevier B.V. All rights reserved.

\section{Introduction}

Heating of a laser material under pumping produces a change of the optical path length (OPL) for a laser radiation propagating through the active medium [1]. This change is highly non-uniform [2], especially for diode-pumping [3,4] with high localization of the heat release [5], so the material can act as a lens-like medium (thermal lens effect) [6]. It is recognized to be an important limitation of power scaling capabilities for solid-state lasers. Thermal lens can lead to the drop of the output power, distortions in the profile of the output beam, depolarization and even laser ceasing [6-8]. Thus, a lot of attention is paid for elimination of thermal lensing. The first route here is the variation of pumping and cooling conditions (the heat removal) [8] and appropriate thermal-lens-insensitive cavity design [9-11]. The second idea is to achieve near-zero change of the optical path length, or athermal behavior [12].

The reason for athermal behavior of the laser materials is related with the fact that OPL is changed due to several effects of different nature $[6,8]$. They are temperature dependence of the refractive index (expressed by the thermo-optic coefficient, $\mathrm{d} n / \mathrm{d} T$ ), photoelastic effect and crystal bulging due to thermal expansion.

\footnotetext{
* Corresponding author. Tel.: + 375(17)2939188; fax: +375(17)2926286.

E-mail address: k.yumashev@tut.by (K.V. Yumashev).
}

The latter term is positive for majority of laser materials, while remaining ones can be either positive or negative $[8,13]$. Thus, partial or even complete compensation of OPL can be achieved. It was realized for Nd and Er glasses by varying of their composition (the glass system) [14]. In principle, this idea can be also extended to an isostructural series of "mixed" crystals. However, investigation of crystals reveals a new way for compensation of OPL and further power scaling of solid-state lasers. It is related to an intrinsic crystal anisotropy, so one can vary the influence of thermal, optical and thermo-optical properties of the crystal on characteristics of the light propagating inside it by appropriate cutting $[12,15]$. Thus, the term "athermal direction" of light propagation was introduced.

A clear example is the double tungstates, DTs, with common formula $\mathrm{X}^{+} \mathrm{R}^{3+}\left(\mathrm{WO}_{4}\right)_{2}$ where $\mathrm{X}=\mathrm{Na}$ or $\mathrm{K}$ and $\mathrm{R}=\mathrm{Gd}$, $\mathrm{Y}$ or $\mathrm{Lu}$. These crystals are excellent hosts for doping with laser-active trivalent rare-earth ions like $\mathrm{Yb}^{3+}$ or $\mathrm{Tm}^{3+}$, that results in the realization of high-efficient diode-pumped solid-state lasers $[16,17]$ (including fs ones [18]). DTs offer (i) large and negative TOCs [12,15], (ii) strong optical anisotropy and (iii) anisotropy of thermal expansion [19]. Thus, all these features can lead to cancellation of OPL changes and provide the desired athermal behavior. From the other hand, monoclinic DTs are optically biaxial, so the solution of this problem is rather complicated. Thus, two routes were proposed in the literature, namely experimental studies of thermal lensing [20-23] and calculations based 
on the material parameters [12,15,24]. First route is limited, as results obtained can be applied only for specific experimental geometry. Second one requires handling with large set of optical, thermal, thermo-optic and elastic properties [5]. Theoretical analysis of the origins of athermal behavior is presented in [25].

In the present paper, we aim to enhance the possibilities of second way, i.e. to perform all-space analysis of existence of athermal directions and their dispersion in monoclinic and biaxial $\mathrm{KY}\left(\mathrm{WO}_{4}\right)_{2}$ crystal, for the first time, to the best of our knowledge. Previous calculations of athermal directions were restricted only to principal planes of the optical indicatrix and one isonormal wave, as well as fixed light wavelength.

\section{Basic equations}

The variation of the OPL in a diode-pumped laser material placed into a laser cavity (or acting as an end mirror in "microchip" resonator) is typically represented by a so-called "generalized" thermo-optic coefficient [5]:

$\chi_{\text {laser }}=(\mathrm{d} n / \mathrm{d} T)_{\varepsilon}+P_{\mathrm{PE}}+(1+\nu)(n-1) \alpha$,

$\chi_{\text {microchip }}=(\mathrm{d} n / \mathrm{d} T)_{\varepsilon}+P_{\mathrm{PE}}+(1+\nu) n \alpha$.

Here $(\mathrm{d} n / \mathrm{d} T)_{\varepsilon}$ is temperature coefficient of the refractive index under constant strain (we will denote it later as $\mathrm{d} n / \mathrm{d} T$ ). Like the refractive index $n$, it depends both on the light propagation direction (expressed by unit vector of phase normal $\boldsymbol{n}$ ) and polarization (expressed by electric displacement vector $\boldsymbol{D}$ ). In addition, both $n$ and $\mathrm{d} n / \mathrm{d} T$ values have their own dispersion. $P_{\mathrm{PE}}$ term represents photoelastic effect (variation of the refractive index due to thermally-induced stresses). The last term corresponds to the microscopic bulging of crystal endfaces due to nonuniform thermal expansion. Here $\alpha$ is the thermal expansion coefficient in the direction of light propagation and $\nu$ is the small parameter related with elastic properties (for isotropic material, it is the Poisson ratio). It shows the contribution of axial strain that is not related with pure thermal expansion. In the present paper, we will reduce the "generalized" thermo-optic coefficient to (following previous papers $[12,15])$ :

$\mathrm{d} n / \mathrm{d} T+(n-1) \alpha=0$,

$\mathrm{d} n / \mathrm{d} T+n \alpha=0$.

In other words, we will neglect the $P_{\mathrm{PE}}$ term and the contribution of the strain that is not related with pure thermal expansion. The reason for this is that these effects are mainly responsible for astigmatism of thermal lens and depolarization losses, as well as they typically do not determine the sign of $\chi$ coefficient $[5,26]$ that is important for the analysis of OPL cancellation. From this point of view, our calculations are eligible for diode-pumped laser engineering. Eq.(2) is called "laser cavity" and "solid-etalon" configuration.

The coordinate frame for all-space description of athermal directions in $\mathrm{KY}\left(\mathrm{WO}_{4}\right)_{2}$ is shown in Fig. 1. It coincides with the right-handled frame of the optical indicatrix with $N_{\mathrm{p}}, N_{\mathrm{m}}$ and $N_{\mathrm{g}}$ axes. The tensor of dielectric permeability $\varepsilon$ has a diagonal form in this frame with three different eigen values, namely $\varepsilon_{\mathrm{p}}=n_{\mathrm{p}}^{2}$, $\varepsilon_{\mathrm{m}}=n_{\mathrm{m}}^{2}, \varepsilon_{\mathrm{g}}=n_{\mathrm{g}}^{2}$. Here $n_{\mathrm{p}}<n_{\mathrm{m}}<n_{\mathrm{g}}$ are the principal refractive indices. In the figure, $\boldsymbol{n}$ is unit vector of phase normal, $\theta$ is the angle between $N_{\mathrm{g}}$ axis and $\boldsymbol{n}$ vector, and $\psi$ is the angle between $N_{\mathrm{p}}$ axis and projection of $\boldsymbol{n}$ into the $p-m$ plane.

Thermal expansion tensor takes diagonal form in its own principal frame, $\left\{X_{i}^{\prime}\right\} . X_{2}^{\prime}$ axis coincides with $N_{\mathrm{p}}$ one, while $X_{1}^{\prime}$ and $X_{3}^{\prime}$ axes are positioned in the $m-g$ principal plane, with the angle

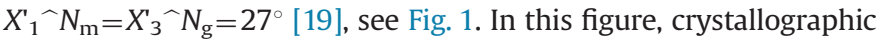
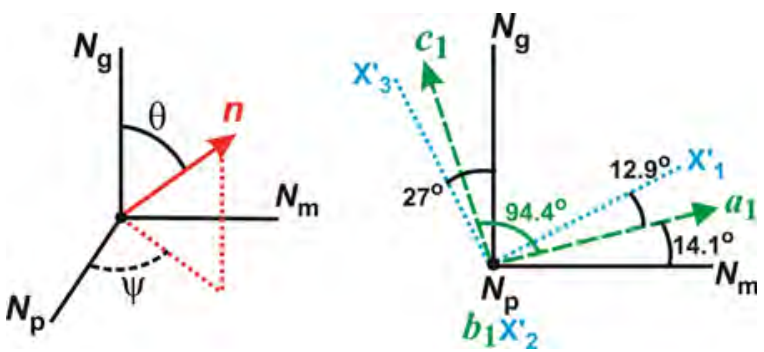

Fig. 1. A coordinate frame chosen for all-space representation of athermal directions (left image); the mutual orientation of principal axes in the $N_{\mathrm{m}}-N_{\mathrm{g}}$ plane (right image).

axes $\boldsymbol{a}_{1}, \boldsymbol{b}_{1}$ and $\boldsymbol{c}_{1}$ (chosen within I2/c crystallographic setting of $C_{2 \mathrm{~h}}^{6}$ space group) are also shown. From the symmetry considerations, the range $0<\psi<\pi / 2$ and $-\pi / 2<\theta<\pi / 2$ is enough for all-space descriptions of athermal direction.

For an arbitrary $\boldsymbol{n}$ vector orientation, two isonormal waves can propagate in a biaxial crystal with mutually orthogonal vectors of electric displacement, $\boldsymbol{D}_{1,2}$, so $\boldsymbol{D}_{1} \boldsymbol{D}_{2}=0$ (in common case, the corresponding electric field vector is not orthogonal, $\boldsymbol{E}_{1} \boldsymbol{E}_{2} \neq 0$ ). These two waves will correspond to different refractive indices $n_{1,2}$. The waves are typically denoted as "fast" $\left(\boldsymbol{D}_{1}\right)$ and "slow" $\left(\boldsymbol{D}_{2}\right)$, meaning higher and lower phase velocity of light propagation in the crystal $\left(n_{1}<n_{2}\right)$. For both of these two waves, conditions of athermal behavior can be satisfied separately. In order to perform the all-space analysis of athermal properties, we consider all possible $\boldsymbol{n}$ directions [the pairs of $(\psi, \theta)$ angles] and calculate the values of $n, \mathrm{~d} n / \mathrm{d} T$ and $\alpha$ accordingly (see below). Then, we select the $\boldsymbol{n}$ orientations that provide compensation of OPL, in accordance with Eqs. (2a) or (2b) (depending on the chosen configuration).

The analytical expression for refractive indices $n_{1,2}$ of "fast" and "slow" waves as a function of $(\psi, \theta)$ angles derived in the present paper is

$$
\begin{aligned}
\frac{1}{n_{1,2}^{2}}= & \frac{1}{n_{m}^{2}}+\frac{n_{m}^{2}-n_{p}^{2}}{2 n_{m}^{2} n_{p}^{2}}\left(\sin ^{2} \psi \sin ^{2} \theta+\cos ^{2} \theta\right)-\frac{n_{g}^{2}-n_{m}^{2}}{2 n_{m}^{2} n_{g}^{2}} \sin ^{2} \theta \\
& \pm \sqrt{\begin{array}{l}
\frac{\left(n_{m}^{2}-n_{p}^{2}\right)^{2}}{4 n_{m}^{4} n_{p}^{4}}\left(\sin ^{2} \psi \sin ^{2} \theta+\cos ^{2} \theta\right)^{2}+\frac{\left(n_{g}^{2}-n_{m}^{2}\right)^{2}}{4 n_{m}^{4} n_{g}^{4}} \sin ^{4} \theta \\
+2 \frac{n_{m}^{2}-n_{p}^{2}}{2 n_{m}^{2} n_{p}^{2}} \frac{n_{g}^{2}-n_{m}^{2}}{2 n_{m}^{2} n_{g}^{2}}\left(\sin ^{2} \psi-\cos ^{2} \psi \cos ^{2} \theta\right) \sin ^{2} \theta
\end{array}}
\end{aligned}
$$

The common method for evaluation of this expression is described in $[27,28]$. Recently, similar problem was also solved in [29]. However, the formula for refractive indices was referred to the angle between $\boldsymbol{E}$ and $\boldsymbol{D}$ vectors, as well as $(\psi, \theta)$, that is not convenient in practical calculations. In the present paper, we present the expression depending only on $(\psi, \theta)$ angles. For the principal planes of the optical indicatrix, Eq. (3) is simplified to

$$
\begin{aligned}
& n_{2}=n_{g} \text { and } \frac{1}{n_{1}^{2}}=\frac{\sin ^{2} \psi}{n_{p}^{2}}+\frac{\cos ^{2} \psi}{n_{m}^{2}}(m-p \text { plane, } \theta=\pi / 2), \\
& n_{1}=n_{p} \text { and } \frac{1}{n_{2}^{2}}=\frac{\cos ^{2} \theta}{n_{m}^{2}}+\frac{\sin ^{2} \theta}{n_{g}^{2}}(m-g \text { plane, } \psi=\pi / 2), \\
& n_{1,2}=n_{m} \text { or } \frac{1}{n_{2,1}^{2}}=\frac{\cos ^{2} \theta}{n_{p}^{2}}+\frac{\sin ^{2} \theta}{n_{g}^{2}}(p-g \text { plane, } \psi=0),
\end{aligned}
$$

For light propagating in the principal plane, one wave with vector $\boldsymbol{D}$ perpendicular to the plane will always have the same refractive index (independent of $\boldsymbol{n}$ ). For another wave with vector $\boldsymbol{D}$ positioned in this plane, the refractive index will depend on the 
light propagation direction. Such waves for biaxial crystals are sometimes called "o-wave" and "e-wave" [27,28], accordingly, as their propagation is similar to the case of uniaxial crystal. Similar notations were introduced also recently in the review paper [30] devoted particularly to the description of anisotropy of optical properties of biaxial monoclinic crystals. It should be noted that the terms "fast" and "slow" wave, from the one hand, and " $o$-wave" and "e-wave", from another, are not identical. The reason for this is the relation between principal refractive indices, $n_{\mathrm{p}}<n_{\mathrm{m}}<n_{\mathrm{g}}$. Indeed

$$
\begin{aligned}
& \text { for } m-p \text { plane "fast" wave }\left(\boldsymbol{D}_{1}\right) \text { is "e-wave", } \\
& \text { "slow" wave }\left(\boldsymbol{D}_{2} \| N_{\mathrm{g}}\right) \text { is "o-wave"; } \\
& \text { for } m-g \text { plane "fast" wave }\left(\boldsymbol{D}_{1} \| N_{\mathrm{p}}\right) \text { is "o-wave", } \\
& \text { "slow" wave }\left(\boldsymbol{D}_{2}\right) \text { is "e-wave"; } \\
& \text { for } p \text {-g plane "fast" wave }\left(\boldsymbol{D}_{1} \| N_{\mathrm{m}}\right) \text { is "o-wave" for } V<\theta<\pi / 2 \text {, } \\
& \text { "fast" wave }\left(\boldsymbol{D}_{1}\right) \text { is "e-wave" for } 0<\theta<V \text {, } \\
& \text { "slow" wave }\left(\boldsymbol{D}_{2} \| N_{\mathrm{m}}\right) \text { is "o-wave" for } 0<\theta<V \text {, } \\
& \text { "slow" wave }\left(\boldsymbol{D}_{2}\right) \text { is "e-wave" for } V<\theta<\pi / 2 \text {. }
\end{aligned}
$$

The most interesting is the case of $p-g$ plane that contains the optical axes $O\left(V\right.$ is the angle between the $O$ axes and $N_{\mathrm{g}}$ principal axis). For this plane, if the $\mathrm{n}$ vector is rotated from $N_{\mathrm{p}}$ axis $(\theta=\pi / 2)$ to $N_{\mathrm{g}}$ axis $(\theta=0)$, the "fast" wave switches from " 0 -wave" to "e-wave". Moreover, if $\mathrm{n}$ is parallel to $O$ directions, both isonormal waves correspond to the same refractive index, namely $n_{\mathrm{m}}$, so the definitions "fast" and "slow" are not applicable. Thus, we use "fast" and "slow" terms for description of arbitrary $\boldsymbol{n}$ orientations; and we use "o-wave" and "e-wave" terms in addition and only for the principal planes, in order to avoid confusion.

The expression for thermo-optic coefficients $\mathrm{d} n_{1,2} / \mathrm{d} T$ for "fast" and "slow" waves is obtained by differentiation of Eq. (3) [the square root is the same as in Eq. (3)]: here $\alpha_{\mathrm{p}}=2.0, \alpha_{\mathrm{m}}=10.8$ and $\alpha_{\mathrm{g}}=17.4$ are the diagonal components of thermal expansion tensor in the optical indicatrix frame and $\alpha_{\mathrm{mg}}=-6.1$ is the non-zero non-diagonal component [19] (the units are $10^{-6} \mathrm{~K}^{-1}$ ).

The description of dispersion of athermal directions requires the information about wavelength-dependence of principal refractive indices, $n_{\mathrm{i}}(i=p, m, g)$, as well as thermo-optic coefficients, $\mathrm{d} n_{\mathrm{i}} / \mathrm{d} T$. In the present paper, we perform our original fitting of Sellmeier formulas based on our own measurements of the refractive indices [24], as well as data presented in [32]. It was motivated by the fact that previously reported formulas cannot adequately describe the position of optical axes in $\mathrm{KY}\left(\mathrm{WO}_{4}\right)_{2}$. The three-term expression for Sellmeier fit is

$n_{i}^{2}=A_{i}+\frac{B_{i}}{1-\left(C_{i} / \lambda\right)^{2}}-D_{i} \lambda^{2}$

here $A_{\mathrm{i}}, B_{\mathrm{i}}, C_{\mathrm{i}}$ and $D_{\mathrm{i}}$ are Sellmeier coefficients, see Table 1 , light wavelength $\lambda$ is expressed in $\mu \mathrm{m}$. Thermo-optic dispersion formulas for $\mathrm{KY}\left(\mathrm{WO}_{4}\right)_{2}$ were recently reported in [33].

\section{Results and discussion}

On the basis of dispersion relations for $n$ and $\mathrm{d} n / \mathrm{d} T$, we calculated wavelength- and temperature-dependent position of the optical axes for $\mathrm{KY}\left(\mathrm{WO}_{4}\right)_{2}$ crystal (Fig. 2), the data that can be useful for the design of a so-called "conical refraction" laser [33] with improved laser performance and tunable polarization state. Such a detailed analysis has not been performed previously. Moreover, calculation of optical axis position is crucial for interpretation of the results on athermal directions. At the wavelength of $1.03 \mu \mathrm{m}$ and at the room temperature (RT), optical axes $O$ are positioned at the angle $V= \pm 43^{\circ} 40^{\prime}$ from $N_{\mathrm{g}}$ axis in the $p-g$ plane.

$$
\begin{aligned}
& \frac{1}{n_{1,2}^{3}} \frac{d n_{1,2}}{d T}=\frac{1}{2 n_{m}^{3}} \frac{d n_{\mathrm{m}}}{d T}\left(1-\sin ^{2} \psi \sin ^{2} \theta\right)+\frac{1}{2 n_{p}^{3}} \frac{d n_{\mathrm{p}}}{d T}\left(\sin ^{2} \psi \sin ^{2} \theta+\cos ^{2} \theta\right)+\frac{1}{2 n_{g}^{3}} \frac{d n_{\mathrm{g}}}{d T} \sin ^{2} \theta \\
& \pm \frac{1}{\sqrt{\cdots}}\left(\begin{array}{l}
\left(\frac{1}{2 n_{p}^{3}} \frac{d n_{p}}{d T}-\frac{1}{2 n_{m}^{3}} \frac{d n_{m}}{d T}\right)\left(\frac{n_{m}^{2}-n_{p}^{2}}{2 n_{m}^{2} n_{p}^{2}}\left(\sin ^{2} \psi \sin ^{2} \theta+\cos ^{2} \theta\right)^{2}+\frac{n_{g}^{2}-n_{m}^{2}}{2 n_{g}^{2} n_{m}^{2}}\left(\sin ^{2} \psi-\cos ^{2} \psi \cos ^{2} \theta\right) \sin ^{2} \theta\right) \\
+\left(\frac{1}{2 n_{m}^{3}} \frac{d n_{m}}{d T}-\frac{1}{2 n_{g}^{3}} \frac{d n_{g}}{d T}\right)\left(\frac{n_{g}^{2}-n_{m}^{2}}{2 n_{g}^{2} n_{m}^{2}} \sin ^{4} \theta+\frac{n_{m}^{2}-n_{p}^{2}}{2 n_{m}^{2} n_{p}^{2}}\left(\sin ^{2} \psi-\cos ^{2} \psi \cos ^{2} \theta\right) \sin ^{2} \theta\right)
\end{array}\right)
\end{aligned}
$$

This is the first derivation of analytical expressions for thermooptic coefficients for an arbitrary light propagation direction in a biaxial crystal. The cases of the principal planes of the optical indicatrix are

$\frac{d n_{2}}{d T}=\frac{d n_{g}}{d T}$ and $\frac{d n_{1}}{d T}=\frac{d n_{p}}{d T} \frac{n_{1}^{3}}{n_{p}^{3}} \sin ^{2} \psi+\frac{d n_{p}}{d T} \frac{n_{1}^{3}}{n_{p}^{3}} \cos ^{2} \psi(m-p$ plane $)$,

$\frac{d n_{1}}{d T}=\frac{d n_{p}}{d T}$ and $\frac{d n_{2}}{d T}=\frac{d n_{m}}{d T} \frac{n_{2}^{3}}{n_{m}^{3}} \cos ^{2} \theta+\frac{d n_{g}}{d T} \frac{n_{2}^{3}}{n_{g}^{3}} \sin ^{2} \theta(m-$ gplane $)$.

$\frac{d n_{1,2}}{d T}=\frac{d n_{m}}{d T}$ and $\frac{d n_{2,1}}{d T}=\frac{d n_{p}}{d T} \frac{n_{2,1}^{3}}{n_{p}^{3}} \cos ^{2} \theta+\frac{d n_{g}}{d T} \frac{n_{2,1}^{3}}{n_{g}^{3}} \sin ^{2} \theta$ ( $p-$ gplane),

The expression for thermal expansion coefficient $\alpha$ in the direction of vector $\boldsymbol{n}$ is [deduced from more common one [31]): $\alpha=\alpha_{p} \sin ^{2} \theta \cos ^{2} \psi+\alpha_{m} \sin ^{2} \theta \sin ^{2} \psi+\alpha_{g} \cos ^{2} \theta+\alpha_{m g} \sin 2 \theta \sin \psi$.
The all-space analysis of athermal behavior of $\mathrm{KY}\left(\mathrm{WO}_{4}\right)_{2}$ for a "laser cavity" configuration is shown in Fig. 3, the light wavelength is $1.03 \mu \mathrm{m}$ (that corresponds to the output wavelength of $\mathrm{Yb}: \mathrm{KY}$ $\left(\mathrm{WO}_{4}\right)_{2}$ lasers). Here blue and red curves represent the position of athermal directions that correspond to two isonormal waves ("fast" and "slow"), bold vertical and horizontal lines - the principal planes of optical indicatrix $(p-g, m-g$ and $m-p$ planes), black points $-N_{\mathrm{p}}$ and $N_{\mathrm{m}}$ principal axes, blue points - the position of two optical axes $\left(\psi=0, \theta= \pm 43.7^{\circ}\right)$ in the $p-g$ plane. In-plane athermal directions can exist only for $m-g$ and $p-g$ planes. Each plane contains four directions, two for $o$-wave and two for $e$-wave. It should be noted that optical axes causes a peculiarity in the

Table 1

Sellmeier coefficients for principal refractive indices of $\mathrm{KY}\left(\mathrm{WO}_{4}\right)_{2}$ crystal at room temperature.

\begin{tabular}{lllll}
\hline$n$ & \multicolumn{3}{l}{ Sellmeier coefficients } \\
\cline { 2 - 5 } & $A$ & $B$ & $C, \mu \mathrm{m}$ & $D, 10^{-3} \mu \mathrm{m}^{-2}$ \\
\hline$n_{\mathrm{p}}$ & 2.69161 & 1.15780 & 0.21270 & 18.815 \\
$n_{\mathrm{m}}$ & 2.52932 & 1.46328 & 0.20629 & 20.927 \\
$n_{\mathrm{g}}$ & 2.39921 & 1.75636 & 0.20075 & 13.263 \\
\hline
\end{tabular}


solutions (see inset in Fig. 3), very similar to the one observed recently for anisotropic absorption of another monoclinic crystal [30]. The remaining $m-p$ plane contains no athermal direction. In common, curves plotted for two possible isonormal waves do not intersect. Thus, each athermal direction is strictly related with a fixed polarization state. However, there exists the possibility for

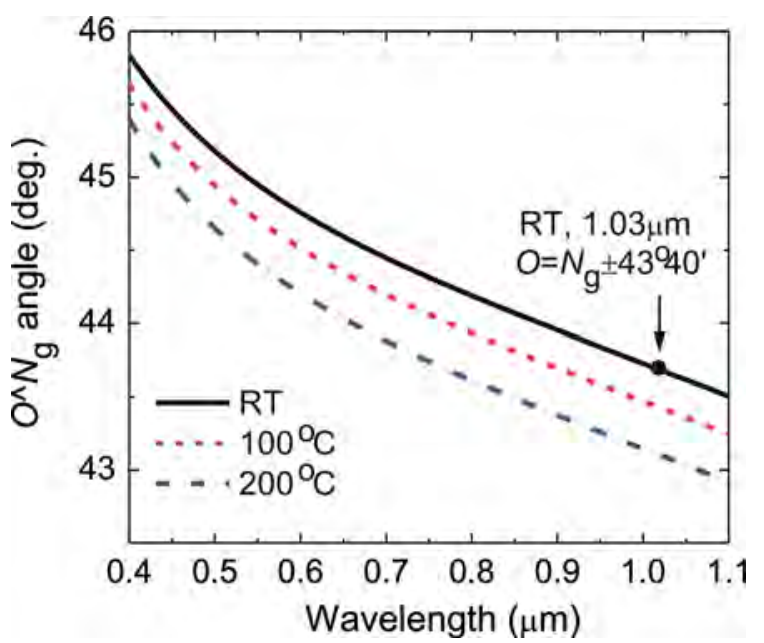

Fig. 2. Dependence of angle between optical axis $O$ and $N_{\mathrm{g}}$ axis (in $p-g$ plane) on the light wavelength plotted for several temperatures (RT is room-temperature), for $\mathrm{KY}\left(\mathrm{WO}_{4}\right)_{2}$ crystal.

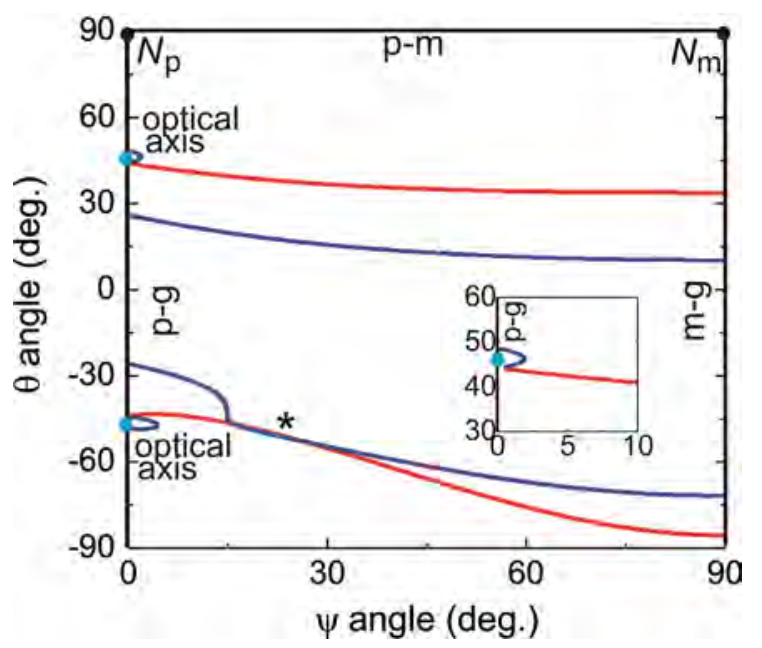

Fig. 3. The all-space analysis of athermal properties for $\mathrm{KY}\left(\mathrm{WO}_{4}\right)_{2}$ crystal: "laser cavity" configuration (at $1.03 \mu \mathrm{m}$ ); inset represents the surrounding of the optical axis. (For interpretation of the references to color in this figure, the reader is referred to the web version of this article.)

Table 2

Location of athermal directions in the principal planes of optical indicatrix for KY $\left(\mathrm{WO}_{4}\right)_{2}$ crystal for two isonormal waves (the light wavelength is $1.03 \mu \mathrm{m}$ ).

\begin{tabular}{|c|c|c|}
\hline \multicolumn{3}{|l|}{ Principal plane } \\
\hline$p-m$ plane $(\theta=\pi / 2)$ & $p-g$ plane $(\psi=0)$ & $m-g$ plane $(\psi=\pi / 2)$ \\
\hline \multicolumn{3}{|c|}{ "Laser cavity" configuration } \\
\hline Not exist & $\begin{array}{l}\theta= \pm 48.9^{\circ}(o) \\
\theta= \pm 29.2^{\circ}(e)\end{array}$ & $\begin{array}{l}\theta=-73.5^{\circ} \text { and } 11.9^{\circ}(o) \\
\theta=-86.2^{\circ} \text { and } 34.5^{\circ}(e)\end{array}$ \\
\hline \multicolumn{3}{|c|}{ "Solid-etalon" configuration } \\
\hline$\psi= \pm 42.0^{\circ}(0)$ & $\theta= \pm 67.1^{\circ}(0)$ & $\theta=55.8^{\circ}$ and $62.6^{\circ}(o)$ \\
\hline$\psi= \pm 39.1^{\circ}(e)$ & $\theta= \pm 58.0^{\circ}(e)$ & not exist $(e)$ \\
\hline
\end{tabular}

some direction of light propagation to be athermal for both waves (marked in Fig. 3 by asterisk). In this separate case there is an interval for $\psi$ angles $\left(15^{\circ} \ldots 35^{\circ}\right)$ for which one can find corresponding angle $\theta$ defining athermal directions for both waves.

The details of in-plane athermal directions are shown in Table 2. For $p-g$ plane, directions $N_{\mathrm{g}} \pm 48.9^{\circ}$ and $N_{\mathrm{g}} \pm 29.2^{\circ}$ are athermal for 0 -wave $\left(\boldsymbol{E} \| N_{\mathrm{m}}\right)$ and $e$-wave, accordingly. Optical axes $\left(O=N_{\mathrm{g}} \pm 43.6^{\circ}\right)$ are near-athermal for $o$-wave, but they are far from athermal for $e$-wave. Thus, although $O$-cut crystals will possess no birefringence at room-temperature, their thermooptic properties will vary with light polarization. As a result, for a so-called "conical refraction" laser [34,35] based on $O$-cut crystal, laser output can be still partially polarized for thermal-lenssensitive cavities. For $p$ - $g$ plane, athermal directions are positioned symmetrically around $N_{\mathrm{g}}$ and $N_{\mathrm{p}}$ axes, as major and minor semiaxes of cross-section of ellipsoid of thermal expansion tensor correspond to these axes.

In contrast, for $m-g$ plane the orientation of such cross-section is determined by $X_{1}^{\prime}$ and $X_{3}^{\prime}$ axes. Thus, position of athermal directions will be not symmetrical with respect to optical indicatrix, namely $X_{3}^{\prime} \pm 42.8^{\circ}\left[N_{\mathrm{g}}-73.5^{\circ}\right.$ and $\left.N_{\mathrm{g}}+11.9^{\circ}\right]$ for 0 -wave $\left(\boldsymbol{E} \| N_{\mathrm{p}}\right)$; $N_{\mathrm{g}}-86.2^{\circ}$ and $N_{\mathrm{g}}+34.5^{\circ}$ for $e$-wave. It should be noted that for Yb-doped DTs, highest gain corresponds to light polarizations $\boldsymbol{E} \| N_{\mathrm{m}}$ and $\boldsymbol{E} \| N_{\mathrm{p}}$ [16]. Thus, for both $p-g$ and $m-g$ planes $o$-wave will be naturally selected in laser cavity without polarizing elements.

All-space analysis of athermal behavior for "solid-etalon" definition is shown in Fig. 4 (the light wavelength is $1.03 \mu \mathrm{m}$ ). In this case, possibilities for canceling of OPL change are wider (as compared with "laser cavity" one), as in-plane athermal directions exist for all three principal planes. There are 4 directions for $p-m$ and $p-g$ planes (for $p-m$ plane, only two are visible from the figure, and two remaining are positioned symmetrically at the angles of $-\psi$, see inset in Fig. 4), two for 0 -wave and two for $e$-wave, and 2 directions for $m-g$ plane (only for $o$-wave). This is related with higher impact of thermal expansion [ $n \alpha$ vs. $(n-1) \alpha]$ on the variation of OPL for this definition. Moreover, in Fig. 4 the curves representing athermal behavior of separate isonormal waves have two intersection points (marked by asterisk). Thus, there again exist some special directions of light propagations that will be athermal for both possible light polarizations.

For $p-m$ plane, the directions $N_{\mathrm{p}} \pm 42.0^{\circ}$ (o-wave, $\boldsymbol{E} \| N_{\mathrm{g}}$ ) and $N_{\mathrm{p}} \pm 39.1^{\circ}$ ( $e$-wave) are athermal. However, due to the anisotropy of the gain, particularly $e$-wave will be generated in such "athermally"-cut $\mathrm{Yb}: \mathrm{KY}\left(\mathrm{WO}_{4}\right)_{2}$ laser (with the laser wavelength around

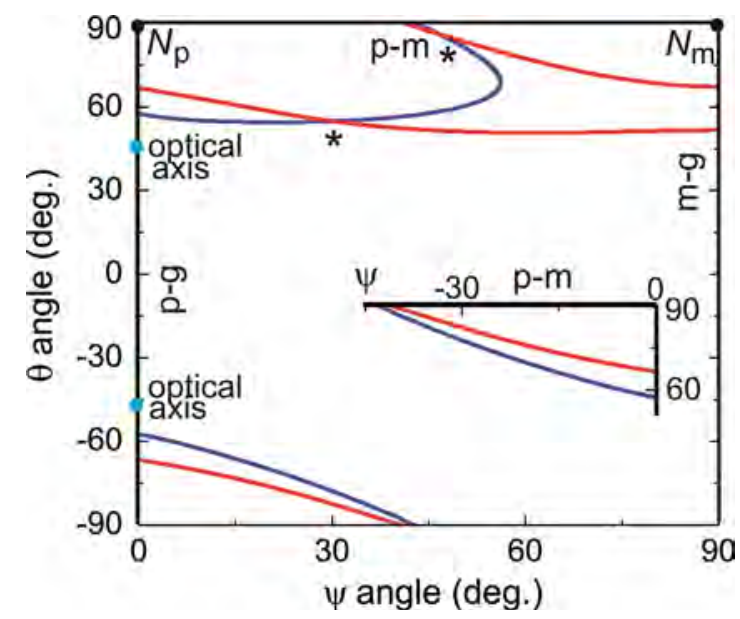

Fig. 4. The all-space analysis of athermal properties for $\mathrm{KY}\left(\mathrm{WO}_{4}\right)_{2}$ crystal: "solidetalon" configuration (at $1.03 \mu \mathrm{m}$ ), inset represents two symmetrical athermal directions in the $p-m$ plane. 

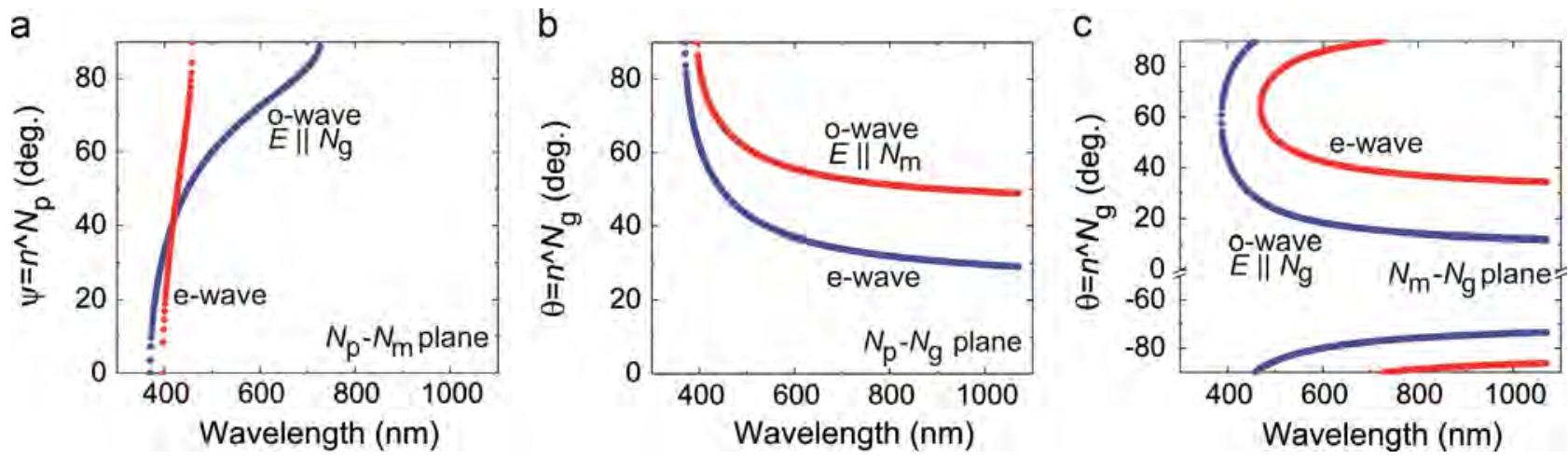

Fig. 5. The dispersion of athermal directions in the principal planes of optical indicatrix: "laser cavity" configuration.

$1030 \mathrm{~nm})$. For $p-g$ plane, the directions $N_{\mathrm{g}} \pm 67.1^{\circ}$ and $N_{\mathrm{g}} \pm 58.0^{\circ}$ are athermal for $o$-wave $\left(\boldsymbol{E} \| N_{\mathrm{m}}\right)$ and $e$-wave, accordingly. For $m-g$ plane, the directions $X_{1}^{\prime} \pm 3.5^{\circ}\left[N_{\mathrm{g}}+55.8^{\circ}\right.$ and $\left.N_{\mathrm{g}}+62.6^{\circ}\right]$ are athermal for 0 -wave $\left(\boldsymbol{E} \| N_{\mathrm{p}}\right)$. As discussed above, for latter two planes, $o$-wave will be naturally-selected in an Yb-laser.

Fig. 5 shows the position of athermal directions for "laser cavity" definition in the principal planes of optical indicatrix as a function of light wavelength. The calculations are performed for visible and near-IR, up to $1.1 \mu \mathrm{m}$, the region of applicability of dispersion formulas for $n$ and $\mathrm{d} n / \mathrm{d} T$ [30]. The consideration of $1 \mu \mathrm{m}$ range is interesting for Yb-lasers. From the other hand, DTs are Raman-active and they are used in visible lasers with subsequent Raman conversion and second-harmonic generation. For all three planes and two possible isonormal waves ( $o$-wave and $e$-wave), there exist some spectral range where one can observe the athermal properties. Typically, this region has a shortwavelength limit in the vicinity of $0.4 \mu \mathrm{m}$. The special case is $p-m$ plane, where a long-wavelength limit of athermal behavior also exists. The dispersion of athermal directions is more pronounced at shorter wavelengths. This is related with higher change of refractive indices and $\mathrm{d} n / \mathrm{d} T$ for the wavelengths approaching UV absorption edge ( $0.27 \mu \mathrm{m}$ for DTs) [33] while thermal expansion is independent on light wavelength. In contrast, in the near-IR the dispersion is weak.

For $p-m$ plane, the region of existence of athermal behavior has both upper and lower limits. Within this range, the athermal directions are right-angle-rotated. This is especially clear for $e$-wave, as $\psi=\boldsymbol{n} \wedge N_{\mathrm{p}}$ is changed from 0 to $\pi / 2$ when the light wavelength is changed from 0.39 to $0.46 \mu \mathrm{m}$. At $0.39 \mu \mathrm{m}$, the crystal cut along the $N_{\mathrm{p}}$ axis is athermal itself (in this case, $e$-wave has the polarization of $\left.\boldsymbol{E} \| N_{\mathrm{m}}\right)$, while at $0.46 \mu \mathrm{m}, N_{\mathrm{m}}$-cut becomes athermal (e-wave corresponds to $\boldsymbol{E} \| N_{\mathrm{p}}$ ). For $e$-wave $\left(\boldsymbol{E} \| N_{\mathrm{g}}\right)$, this range is higher, namely $0.37-0.73 \mu \mathrm{m}$. At the spectral limits, the directions of $N_{\mathrm{p}}$ and $N_{\mathrm{m}}$ axes will be athermal.

In the $p-g$ plane, the dependence of position of athermal directions on the light wavelength is qualitatively different. Indeed, the athermal behavior arises at $0.37-0.4 \mu \mathrm{m}$ for $e$ - and $o$-waves, accordingly (starting from $N_{\mathrm{p}}$ axis). Then, the orientation of athermal directions is stabilized at some value in the near-IR. A similar picture is observed for $m-g$ plane. Here the shortwavelength limit for athermal properties is between 0.39 and $0.47 \mu \mathrm{m}$ for $o$ - and $e$-waves accordingly (starting from the direction of $X_{1}^{\prime}$ axis or in our coordinate system, $N_{\mathrm{g}}+59.1^{\circ}$ ). In Fig. 5(c), the range $-\pi / 2<\theta<\pi / 2$ is shown, as athermal directions are located non-symmetrical with respect to the optical indicatrix axes (as discussed above). Thus, one can see all 4 branches of solutions. In Fig. 5(a and b), two remaining symmetrical branches are not shown, they can be obtained simply by the transformation $\psi \rightarrow-\psi$ for $p-m$ plane or $\theta \rightarrow-\theta$ for $p-g$ plane. The possibilities for
Table 3

Light wavelength providing coincidence of athermal directions with the optical indicatrix axes for $\mathrm{KY}\left(\mathrm{WO}_{4}\right)_{2}$ (the light polarization is denoted in brackets).

\begin{tabular}{lll}
\hline Principal axis & & \\
\hline$p$-axis & $m$-axis & g-axis \\
\hline "Laser cavity" configuration & & \\
$0.40 \mu \mathrm{m}\left(\boldsymbol{E}_{\mathrm{m}}\right)$ & $0.73 \mu \mathrm{m}\left(\boldsymbol{E}_{\mathrm{g}}\right)$ & \\
$0.37 \mu \mathrm{m}\left(\boldsymbol{E}_{\mathrm{g}}\right)$ & $0.46 \mu \mathrm{m}\left(\boldsymbol{E}_{\mathrm{p}}\right)$ & Not exist \\
"solid-etalon" configuration & & \\
$0.44 \mu \mathrm{m}\left(\boldsymbol{E}_{\mathrm{m}}\right)$ & Not exist & \\
$0.39 \mu \mathrm{m}\left(\boldsymbol{E}_{\mathrm{g}}\right)$ & & \\
\hline
\end{tabular}

principal axes of optical indicatrix to coincide with the athermal directions are shown in Table 3. These results do not agree with previous conclusions ([15] and Refs. therein) that $p, m, g$ axes itself cannot be athermal. This is because the analysis in [15] was restricted to only one light wavelength.

The analysis of dispersion of athermal directions for "solidetalon" definition is shown in Fig. 6. In this case, the athermal directions in all three principal planes has only short-wavelength limit of existence (for 0 - and $e$-waves) when (for the exception of $m-g$ plane) they coincide with the optical indicatrix axes, see Table 3. Results from Figs. 5 and 6 significantly extend the idea about athermal behavior of DTs. In the previous papers, athermal directions were calculated at fixed wavelength, only for principal planes of optical indicatrix and 0 -wave. As a result, it was concluded that such directions cannot exist in principle for several principal planes or light polarizations $[15,19]$. However, it is not confirmed, if we consider dispersion of athermal directions and existence of two isonormal waves in the biaxial crystal.

\section{Conclusions}

The analytical expressions for thermo-optic coefficients $\mathrm{d} n / \mathrm{d} T$ for "fast" and "slow" light waves propagating along the arbitrary direction in a biaxial crystal are derived. On the basis of these equations, the analysis of existence of athermal properties is performed for monoclinic and optically biaxial $\mathrm{KY}\left(\mathrm{WO}_{4}\right)_{2}$ at $1.03 \mu \mathrm{m}$ for arbitrary light propagation direction in the crystal (not restricted to principal planes), considering both possible isonormal waves, as well as "laser cavity" and "solid-etalon" definitions. In addition, the dispersion of athermal directions is analyzed for visible and near-IR, based on evaluated three-term Sellmeier formula for refractive indices $n_{\mathrm{p}}, n_{\mathrm{m}}$ and $n_{\mathrm{g}}$. The appearance of directions that can be athermal for both isonormal waves is predicted. The existence of short- and long-wavelength 

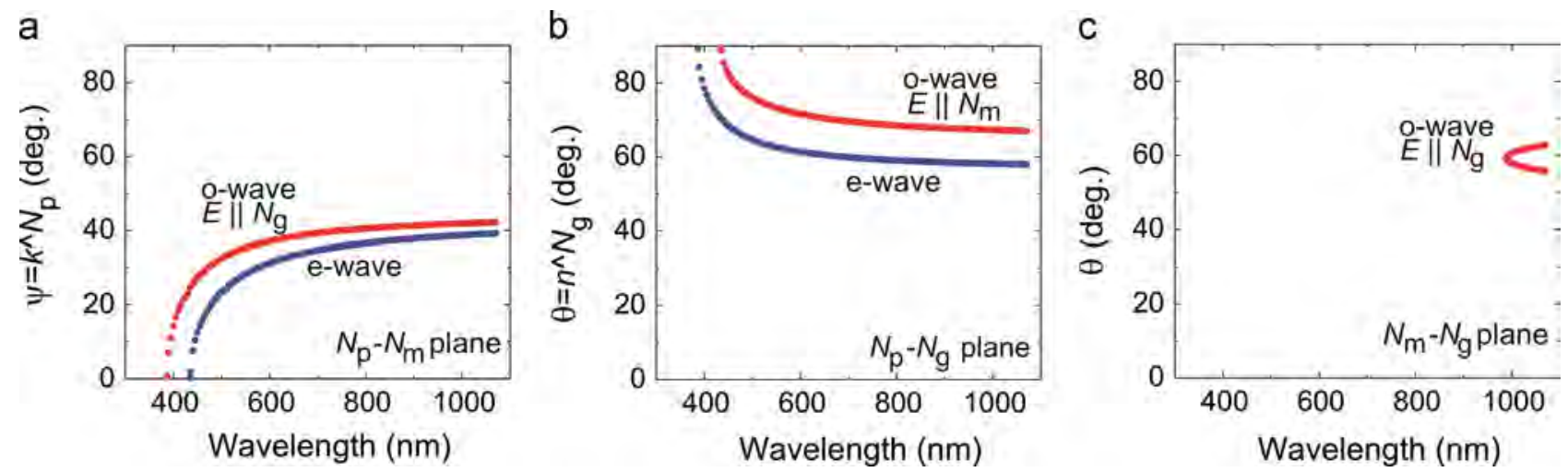

Fig. 6. The dispersion of athermal directions in the principal planes of optical indicatrix: "solid-etalon" configuration.

dispersion limits for athermal behavior and the possibility for optical indicatrix axes to be athermal themselves is shown for the first time for $\mathrm{KY}\left(\mathrm{WO}_{4}\right)_{2}$. Wavelength- and temperature-dependent position of optical axes for $\mathrm{KY}\left(\mathrm{WO}_{4}\right)_{2}$ crystal is also determined.

\section{References}

[1] W.A. Clarkson, J. Phys. D 34 (2001) 2381.

[2] B. Bendow, P.D. Gianino, Appl. Opt. 12 (1973) 710.

[3] U. Farrukh, A.M. Buoncristiani, C.E. Byvik, IEEE J. Quantum Electron. 24 (1988) 2253.

[4] M.E. Innocenzi, H.T. Yura, C.L. Fincher, R.A. Fields, Appl. Phys. Lett. 56 (1990) 1831.

[5] A.K. Cousins, IEEE J. Quantum Electron. 28 (1992) 1057.

[6] W. Koechner, Solid-State Laser Engineering, 6th ed, Springer, New York, 2006.

[7] N. Hodgson, H. Weber, Optical Resonators: Fundamentals, Advanced Concepts and Applications, Springer, London, 1997.

[8] S. Chenais, F. Druon, S. Forget, F. Balembois, P. Georges, Prog Quantum Electron. 30 (2006) 89.

[9] Y. Chen, T.M. Huang, C.F. Kao, C.L. Wang, S.C. Wang, IEEE J. Quantum Electron. 33 (1997) 1424.

[10] M.P. MacDonald, T.h. Graf, J.E. Balmer, H.P. Weber, Opt. Commun 178 (2000) 383.

[11] D. Metcalf, P. Giovanni, J. Zachorowski, M. Leduc, Appl. Opt. 26 (1987) 4508.

[12] S. Biswal, S.P. O'Connor, S.R. Bowman, Appl. Opt. 44 (2005) 3093.

[13] G. Ghosh, E. Palik, Handbook of Thermo-Optic Coefficients of Optical Materials with Applications, Academic Press, San Diego, 1998.

[14] See, for example 〈http://www.kigre.com/glass.html $\rangle$.

[15] S. Vatnik, M.C. Pujol, J.J. Carvajal, X. Mateos, M. Aguiló, F. Díaz, V. Petrov, Appl, Phys. B 95 (2009) 653.
[16] V. Petrov, M.C. Pujol, X. Mateos, O. Silvestre, S. Rivier, M. Aguilo, R.M. Sole, J. Liu, U. Griebner, F. Diaz, Laser Photonics Rev. 1 (2007) 179.

[17] A.A Lagatsky, N.V Kuleshov, V.P Mikhailov, Opt. Commun. 165 (1999) 71.

[18] S. Pekarek, C. Fiebig, M.C. Stumpf, A.E.H. Oehler, K. Paschke, G. Erbert, T. Sudmeyer, U. Keller, Opt. Express 18 (2010) 16320.

[19] P.A. Loiko, K.V. Yumashev, N.V. Kuleshov, G.E. Rachkovskaya, A.A. Pavlyuk, Opt. Mater. 34 (2011) 23.

[20] I.V. Mochalov, Opt. Eng. 36 (1997) 1660.

[21] J.E. Hellstrom, S. Bjurshagen, V. Pasiskevicius, Appl. Phys. B. 83 (2006) 55.

[22] F. Hoos, S. Li, T.P. Meyrath, B. Braun, H. Giessen, Opt. Express 16 (2008) 6041.

[23] P.A. Loiko, K.V. Yumashev, N.V. Kuleshov, V.G. Savitski, S. Calvez, D. Burns, A.A. Pavlyuk, Opt. Express 17 (2009) 23536.

[24] V.V. Filippov, N.V. Kuleshov, I.T. Bodnar, Appl. Phys. B 87 (2007) 611.

[25] R. Moncorgé, O.N. Eremeykina, J.L. Doualan, O.L. Antipov, Opt. Commun. 281 (2008) 2526

[26] P.A. Loiko, V.G. Savitski, A. Kemp, A.A. Pavlyuk, N.V. Kuleshov, K.V. Yumashev, Laser Phys. Lett. 11 (2014) (055002-1-7).

[27] V.V. Filippov, Appl. Opt. 52 (2013) 4377.

[28] F.I. Fedorov, V.V. Filippov, Reflection and refraction of light by transparent crystals, Nauka i Tekhnika, Minsk, 1976 (in Russian).

[29] C. Hernández-Rodríguez, A.B. Fragoso-López, Opt. Laser Technol. 55 (2014) 1.

[30] Y. Petit, S. Joly, P. Segonds, B. Boulanger, Laser Photonics Rev. 7 (2013) 920.

[31] A.J.F. Nye, Physical Properties of Crystals, Clarendon Press, Oxford, 1957.

[32] A.A. Kaminskii, A.F. Konstantinova, V.P. Orekhova, A.V. Butashin, R.F. Klevtsova, A.A. Pavlyuk, Crystallogr. Rep. 46 (2001) 665

[33] P.A. Loiko, K.V. Yumashev, N.V. Kuleshov, G.E. Rachkovskaya, A.A. Pavlyuk, Opt. Mater. 33 (2011) 1688.

[34] A. Abdolvand, K.G. Wilcox, T.K. Kalkandjiev, E.U. Rafailov, Opt. Express 18 (2010) 2753.

[35] J. Hellstrom, H. Henricsson, V. Pasiskevicius, U. Bunting, D. Haussmann, Opt. Lett. (2007) 2783. 